\title{
Picture-Based Multiple-AntennaTechnique for the DVB-T2 Receiver
}

\author{
Budi Setiyanto, Risanuri Hidayat, I Wayan Mustika, and Sunarno
}

\begin{abstract}
Deep-fading can decline the quality of the received signal to below threshold, and interrupts the reception-success (generating an outage or time-out). In DVB-T2 (Digital Video Broadcasting Terrestrial Second Generation) receiver, such interruption can be identified from the displayed-picture. Multiple-antenna is one of techniques to mitigate such problem. This paper presents a multiple-antenna method for receiving the DVB-T2 signal. At any time, one of antennas is selected where the selection action is controlled by the recovered-picture. In case of using two antennas, field-measurement was conducted to collect the real data, later used in the simulation of the proposed algorithm. The result showed its capability to increase the portion of cumulative reception-success duration up to approximately 1.53 times with respect to its equivalent single-antenna.
\end{abstract}

Keywords-DVB-T2, multiple-antenna, picture, receivingsystem, outage, time-out.

\section{INTRODUCTION}

$\mathbf{T}$ HERE are many standards of digital terrestrial television (DTT) broadcasting technology [1], one of which is DVBT2 [2]. This standard applies OFDM (orthogonal frequency division multiplexing) modulation, which is also used by DVB-T and ISDB-T (Integrated Service Digital Broadcasting - Terrestrial) standards [1]. Several countries have adopted DVB-T2 and migrate from analog one to it. Fieldmeasurement on signal-strength distribution [3] and field-trial especially for guiding parameters setting at the transmittingside [4] have been carried out as well.

One of problems in terrestrial radio communication system is related to the multipath propagation effect [5], especially in a mobile environment in which it can decline the received signal quality to below threshold. In DVB-T2, signal with too low quality causes a failed-reception though with the sufficiently high intensity. DVB-T2 receivers produce a moving or still picture, dependent upon its reception whether it is successful or failed. Failed-reception in this case is similar to a link outage (time-out) phenomenon in a microwave transmission system.

One of techniques to mitigate this effect is by using more than one piece of antenna at the receiving-end. For ISDB-T system, a joint AA-CD (adaptive antenna array and carrier diversity) combining technique has been implemented and evaluated using field-experiment [6]. For DVB-T2 reception in

Budi Setiyanto, Risanuri Hidayat, I Wayan Mustika are with the Departement of Electrical Engineering and Information Techology, Faculty of Engineering, Universitas Gadjah Mada, Yogyakarta, Indonesia (e-mail: \{budi_setiyanto, risanuri, wmustik\}@ugm.ac.id,

Sunarno is with the Departement of Engineering Physics and Nuclear Engineering, Faculty of Engineering, Universitas Gadjah Mada, Yogyakarta, Indonesia (e-mail: sunarno@ugm.ac.id). high-speed train, a beamforming receiver based technique has been evaluated using a computerized simulation [7].

In some previous works, every piece of antenna was cascaded to one piece of radio-frequency (RF) unit. Each RFunit converted the signal into baseband (BB). Further signal processing was carried-out at the $\mathrm{BB}$ stage. In designing the future versions of receivers, those techniques could be adopted. However, they are not practically applied in several versions of legacy receivers. A suitable technique applicable for all versions of receiver (legacy and future) therefore is deemed necessary.

This paper presents a proposed multiple-receive-antenna method for the DVB-T2 (Digital Video Broadcasting Terrestrial Second Generation) receivers. At any time, one of antennas is selected for feeding an RF signal to the receiver where a selection action is controlled by the recovered-picture. For this, only one piece of RF-unit is required to support all pieces of antenna. The proposed method is applicable not only for the current but also for the future versions of receiver.

This paper is organized as follows. After introduction in Section I, Section II briefly reviews the multiple-receiveantenna technique. The proposed method is explained in Section III, followed by its research method in Section IV. The analysis of the result is presented in Section V and VI, and finally, it is concluded in Section VII.

\section{DVB-T2 AND MultiPle-ANTENNA RECEIVING SysteMS}

\section{A. DVB-T2 Receiver}

A number of programs carried out in one television (TV) frequency channel, as wide as $8 \mathrm{MHz}$ for example, is only one in analog system, but it is possible more than one in DVB-T2. At the transmitting-side, several programs can be multiplexed into a PLP (physical layer pipe). Several PLPs can further be re-multiplexed to build a multiple-PLP frame format. Finally, this format is transmitted using the OFDM modulation to occupy its frequency channel.

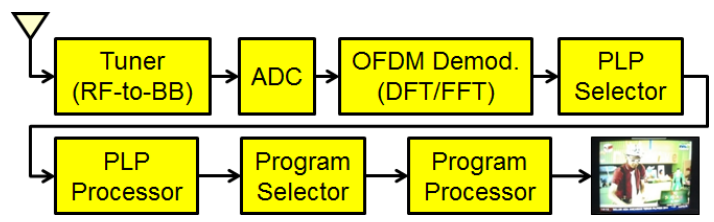

Fig. 1. Simplified block diagram of the DVB-T2 receiver.

Figure 1 presents the simplified block-diagram of the DVBT2 receiver where DFT/FFT (discrete/fast Fourier transform) is used in demodulating the OFDM signal. Tuner in this figure involves all stages from $\mathrm{RF}$ to $\mathrm{BB}$. ADC (analog-to-digital converter) converts the $\mathrm{BB}$ signal to a digital format. PLP processing consists of QAM (quadrature amplitude modulation) demaping, deinterleaving, and FEC (forward error 
control) decoding. Meanwhile, MPEG (moving picture expert group) decoding is included in the program processor block.

\section{B. General Principles of Multiple-Receive-Antenna System}

Figure 2 presents a comparison between single-antenna and multiple-antenna receiving systems. A conventional or singleantenna reception technique can be modelled in Fig. 2 (a) in which a piece of antenna feeds RF signal to a receiver. The receiver can be separated into two stages: front and back stages. It can also be viewed for having only a signal-flow unit. In a TV system, the final output of the receiver is the recovered picture watched by the user.

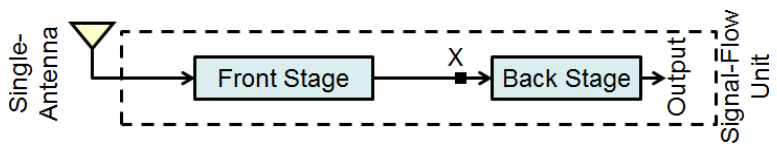

(a)

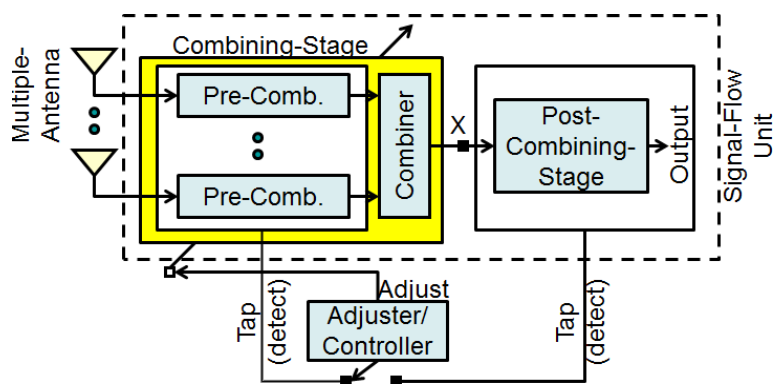

(b)

Fig. 2. Receiving systems: (a) single-antenna, and (b) multiple-antenna

In multiple-antenna system, as shown in Fig. 2 (b), receiver is fed by many pieces of antenna. Front-stage in the singleantenna system is substituted by a combining-stage, and an additional adjuster (controller) unit needs to be provided as well. Combining-stage consists of pre-combiner(s) followed by a combiner. The back-stage in Fig. 2 (a) becomes the postcombining-stage in Fig. 2 (b).

At the combining-stage, RF signals coming from all antennas are processed to construct the signal fed to the next stage (postcombining-stage). The simplest combining process is, of course, by just selecting one of signals entering the combiner.

Combining-stage is activated by a signal (command) generated by the adjuster (controller) unit. To generate this signal (command), this unit monitors or detects (taps) a quantity from either the pre or post combiner stage. In other words, this detected quantity can be viewed as the adjusting (controlling) quantity and should sufficiently represent the final output of the receiver. Therefore, the correlation between it and the receiver output (recovered picture in this case) is highly required.

\section{Multiple-Receive-Antenna for OFDM-Based System}

In OFDM-based receivers, combining operation can be carried-out at the RF [8]-[10], pre-DFT [7], [11]-[16], or postDFT [6], [7], [10]-[12], [15]-[19] stages, or even by jointing some of them [6], [7], [10]-[12], [14], [16]. Several techniques have been experimentally evaluated for DVB-T [19] or ISDB$\mathrm{T}[6],[9],[16]$.

\section{Multiple-Receive-Antenna for DVB-T2}

In DVB-T2 system, various transmit-parameters in conjunction with OFDM and PLP are presettable or selectable.
OFDM parameters involve FFT-size, guard interval (GI) and pilot pattern.

In the beamforming method [7], signal processing algorithm forming two main beams is employed at the pre-DFT stage. This process produces one signal per beam to be passed to the DFT processor. The output signals from all DFT processors are summed-up to construct the final combined signal.

\section{PROPOSED METHOD}

\section{A. Probability Concept Background}

Deep fading can degrade the received signal drop to below threshold. It also interrupts the success of the reception (outage or time-out). Let $I>1$ receiving-antennas is used. If the failure probability of antenna $\# i$ is $f_{i}$, then the probability that all antennas simultaneously fail to receive is $f$, where

$$
\left(\prod_{i=1}^{I} f_{i}\right) \leq f \leq f_{i}, i=1,2,3, \ldots, I
$$

This equation shows that the higher the number of antennas, the lower the failure probability, provided that among antennas are as uncorrelated as possible.

\section{B. Model and Algorithm}

Multiple-antenna technique (method) proposed in this research is modelled in Fig. 3 (a). Antenna \#i provided signal $x_{i}(t)$. At any time, signal fed to receiver, $x(t)$, was selected from one of all antennas. Antenna being selected was, of course, the one being successfully receiving. Reception-success was identified from the monitor, $p(t)$, whether it produced an moving or still picture.

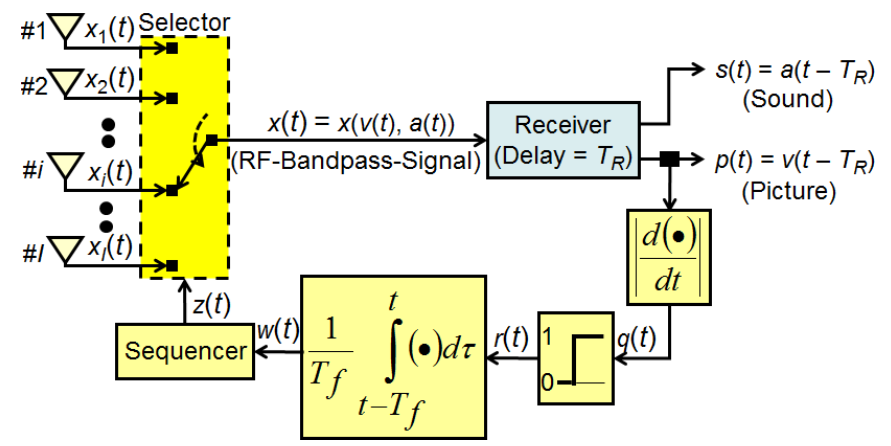

(a)

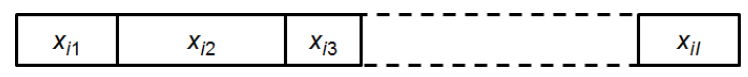

(b)

Fig. 3. The conceptual proposed method: (a) model, and (b) timing diagram illustration of signal fed to receiver.

Receiver input signal, $x(t)$, is an RF-bandpass-signal carrying video and audio signals. It can be expressed as

$$
x(t)=x(v(t), a(t))
$$

where $v(t)$ and $a(t)$ are the video and audio signals, respectively. Extracting this signal to produce picture and sound outputs, $p(t)$ and $s(t)$, respectively, generated a delay as long as $T_{R}$, dependent upon the receiver technology. As an illustration, in some types of receiver, it can achieve several seconds when its selected tuning frequency channel or just its selected program on same channel is changed by the watcher. Considering this, the picture and sound outputs can be 
modelled as the delayed versions of their associated video and audio signals, respectively, or

$$
p(t)=v\left(t-T_{R}\right)
$$

and

$$
s(t)=a\left(t-T_{R}\right)
$$

Motion detection was carried-out in two steps. Firstly, differentiating $p(t)$ regarding time and taking its absolute value of the result to obtain

$$
q(t)=\left|\frac{d p(t)}{d t}\right|
$$

where $q(t) \neq 0$ indicates that motion exists (reception is successful). Secondly, quantizing this absolute value to generate a quantity representing the reception-successfulness

$$
r(t)=\left\{\begin{array}{l}
1, q(t) \neq 0 \\
0, \text { otherwise }
\end{array}\right.
$$

where $r(t)=1$ states that reception is successful.

When reception started failing, control mechanism waited to check whether it was a real or just a spurious failed, for example due to the change of frequency channel or program as illustrated above. This waiting duration, $T_{f}$, must be longer than the receiver delay, $T_{R}$. To do so, $r(t)$ was integrated as long as $T_{f}$ to form

$$
w(t)=\frac{1}{T_{f}} \int_{t-T_{f}}^{t} r(\tau) d \tau
$$

where $w(t)=0$ indicates that a failed condition has really occurred, and switching-over to other antenna is required. Therefore, the switching-over (controlling) rule can be formulated as

$$
x(t)=\left\{\begin{array}{l}
x_{i}(t), w(t) \neq 0 \\
x_{j}(t), \text { otherwise }
\end{array}\right.
$$

where

$$
j=[(i+J) \bmod (I+1)]+1
$$

with $J$ is an integer relying upon the sequencing strategy. Switching-over occurrence was actuated by a command signal, $z(t)$, generated by the sequencer.

Signal fed to receiver was constructed by sequentiallycomposing several time-segments of windowed $x_{i}(t)$ from various $i$-s, as illustrated by a timing-diagram in Fig. 3 (b). Therefore

$$
x(t)=\sum_{i w=1} x_{i w}(t)
$$

with the new subscript "iw" can take any value among 1, 2, 3, ..., and $I$. As example of an extreme case, if the selected signal has never switched-over from antenna \#1, then iw has also never changed from 1 .

For a certain $i$, signal $x_{i}(t)$ provided a successful-reception at several time-segments and failed-reception at others. Similar case can be given by a signal fed to receiver, $x(t)$.

According to the explanation above, setting parameters for the proposed method include: (1) tolerable failed-duration before switch-over $\left(T_{f}\right)$, (2) sequencing strategy $(J)$, and (3) number of antennas $(I)$. In practice, $T_{f}$ needs to adapt to $T_{R}$ of the receiver type used by the watcher. Consequently, common (inexpert) users (TV watchers) need adjusting it by themselves.

\section{Suitability for Legacy and Future Receivers}

$\mathrm{RF}$ aerial input is the only port available in the simplest type of legacy receivers. Such receivers can therefore apply the proposed method where the additional parts in Fig. 3 (a) (from picture detector to selector) are installed as an external unit. In designing a version of future receivers, the proposed method could also be embedded as an internal part.

\section{Hardware Realization}

According to a definition of analog circuit [20], conversion processes in equation (5), (6), and (7) could be realized by using such circuits, whereas the sequencer part generating $z(t)$ from $w(t)$ in Fig. 3 (a) might be practically implemented as a finite-state machine or sequential digital circuit. Further discussion of the hardware realization is beyond the scope of this paper.

\section{Research Method AND the Primary Data}

The performance of the proposed method was compared firstly to its equivalent single-antenna receiving system. It was then followed to its upper-bound or optimistic benefit. It was evaluated by using a simulation in which the simulated-data was collected from real field-measurement - not from the computerized (numerical) simulation.

\section{A. Formulating the Equivalent Single-Antenna System}

Suppose that each antenna in Fig. 3 (a) independently feeds its own receiver, as illustrated in Fig. 4. Therefore, it constructs $I$ independent single-antenna receiving systems. Corresponding quantity representing the success of each antenna, $r_{i}(t)$, is defined similar to $r(t)$ in equation (3).

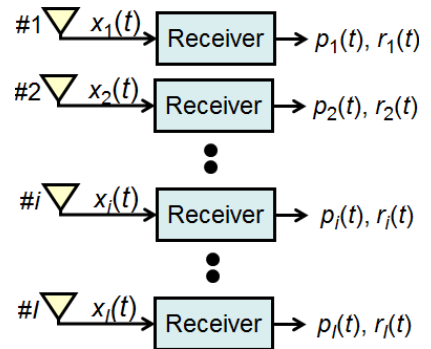

Fig. 4. I sets of single-antenna receiving system.

The cumulative-duration of successful-reception was individually obtained by using antenna $\# i$ evaluated in a period as long as $T_{e v}$ is

$$
L_{i}=\int_{T_{e \nu}} r_{i}(\tau) d \tau
$$

with average

$$
L=\frac{1}{I} \sum_{i=1}^{I} L_{i}
$$

The best antenna is, of course, the one giving the highest $L_{i}$. An antenna might be better or worse than others in receiving one or several TV channels in one or several locations, and vice versa for other(s) channel(s) or location(s). If only one 
antenna is used for all channels in all locations, it cannot be predetermined which antenna is the best choice. Consequently, performance of the proposed method should be compared to every single-antenna counterpart belong to all antennas, and then averaging the result. Therefore, if $L_{i}$ represents the result that belongs to antenna \#i, then $L$ in equation (12) can be interpreted as the result that belongs to its equivalent singleantenna system counterpart.

\section{B. Defining the Optimistic Benefit}

By handling $r_{i}(t)$ as a logic-variable, applying "OR" and "AND" Boolean operations to it gives two auxiliary variables

$$
u(t)=r_{1}(t)+r_{2}(t)+r_{3}(t)+\ldots+r_{I}(t)
$$

and

$$
c(t)=r_{1}(t) r_{2}(t) r_{3}(t) \ldots r_{I}(t)
$$

In equation (13), $u(t)=1$ represents that at least one antenna is successful at that time, whereas $c(t)=1$ in equation (11) represents that all antennas simultaneously (coincidently) pass to receive. Handling $u(t)$ and $c(t)$ as the usual algebraic variables and integrating them as in equation (11) has given two cumulative-durations, notated by $U$ and $C$, respectively.

A proper selection would provide a successful-reception when at least one antenna pass to receive. Therefore, $U$ represents the probably achieved cumulative-duration of successful-reception by the proposed method. An increment of cumulative-duration from $L$ in the single-antenna system up to $U$ in the proposed method is expectable. Therefore, $U / L$ can be viewed as the optimistic-gain or the probably-achieved gain.

When all antennas simultaneously (coincidently) pass to receive, then effectively only one of them is required at that moments, whereas the remainder $I-1$ ones just acts as redundant. In such case, $C=U$, or $C / U=1$. On the other hand, when all antennas perfectly complement to each other, no coincidence occurs. In this case, $C=0$, or $C / U=0$, provided that $U \neq 0$. Therefore, $C / U$ is suitable for representing intersignal correlation level of the radio channel.

\section{Determining the System Performance}

Substituting $r_{i}(t)$ in equation (11) by $r(t)$ provided the cumulative-duration of successful-reception obtained by the proposed method, $S$. If antenna $\# i$ contributes $S_{i}$, then

$$
S=\sum_{i=1}^{I} S_{i}
$$

Let $x^{w}{ }_{i}(t)$ is $x_{i w}(t)$ in Fig. 3 (b) belong to $x_{i}(t)$, and its corresponding reception-success according to equation (6) is $r^{w}{ }_{i}(t)$. If $r_{i}(t)$ in equation (11) is substituted by $r_{i}^{w}(t)$, then the result is $S_{i}$

In this paper, success-rate or success-ratio $(S R)$ is defined as the portion of successful-reception cumulative-duration. If $L / T_{e v}$ is $S R$ of the equivalent single-antenna system, then $U / T_{e v}$ and $S / T_{e v}$ are that of optimistic and factual multiple-antenna system, respectively.

The performance of the proposed method was compared to its optimistic bound and equivalent single-antenna counterpart. Therefore, the most important performance indicators included $S / L$ and $S / U$, where $S / L$ refers to the factual-gain or the achieved-gain.

\section{Running the Simulation}

In simulation, continuous-time operations were converted to discrete-time version by simply substituting $t, T_{f}$, and $T_{e v}$ with integer numbers $k, N$, and $K$, respectively. Omitting $T$ in a written expression converted the continuous-time quantities to their corresponding sequences (series). Some of them include:

$$
\begin{aligned}
& q(k)=p(k)-p(k-1) \\
& w(k)=\frac{1}{N} \sum_{n=1}^{N} r(k-n)
\end{aligned}
$$

and

$$
L_{i}=\sum_{k=1}^{K} r_{i}(k)
$$

Other quantities were similarly obtained by simply replacing the absolute time, $t$, by its corresponding sequence index, $k$. Simulator needs, of course, data completely informing the $p_{i}(k)$ for all $i$-s. In this research, such data was obtained from fieldmeasurement.

\section{E. Preparing Data for Simulation}

Field-measurement for supplying data to simulator was carried-out only for a dual-antenna system, or $I=2$. It provided $p_{1}(k)$ and $p_{2}(k)$ of Fig. 4. Field-measurement work is illustrated in Fig. 5. Two sets (\#1 and \#2) of independent single-antenna receiving system were installed in an automobile. The automobile travelled several route-segments. At each routesegment, pictures $\left(p_{1}(t)\right.$ and $\left.p_{2}(t)\right)$ displayed by their monitors were recorded by a common video camera. Result of this work was one $K T$-second duration video file per route-segment.

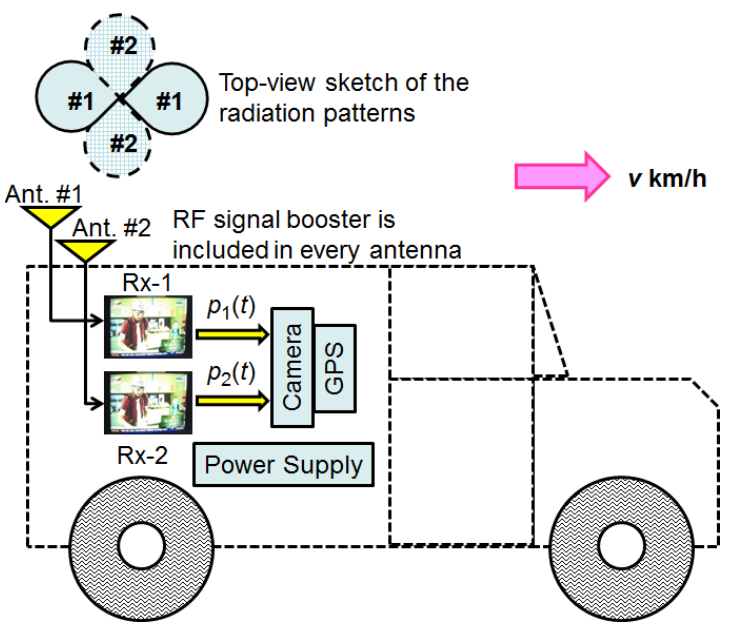

Fig. 5. Illustration of field-measurement (data collection and recording) work.

For the sake of its simplicity, this research did not evaluate the effect of antenna's characteristics or placements. However, they should be considered to obtain a feasible result. Antenna \#2 was oriented perpendicular to and placed slight below antenna \#1. Such arrangement was expected to give a radiation pattern as shown in the top-side of Fig. 5. Antenna \#1 was expected to receive signals arrived mainly from directions parallel to automobile movement, whereas signals arriving from directions perpendicular to it were expected to be received by antenna \#2. 
PICTURE-BASED MULTIPLE-ANTENNATECHNIQUE FOR THE DVB-T2 RECEIVER

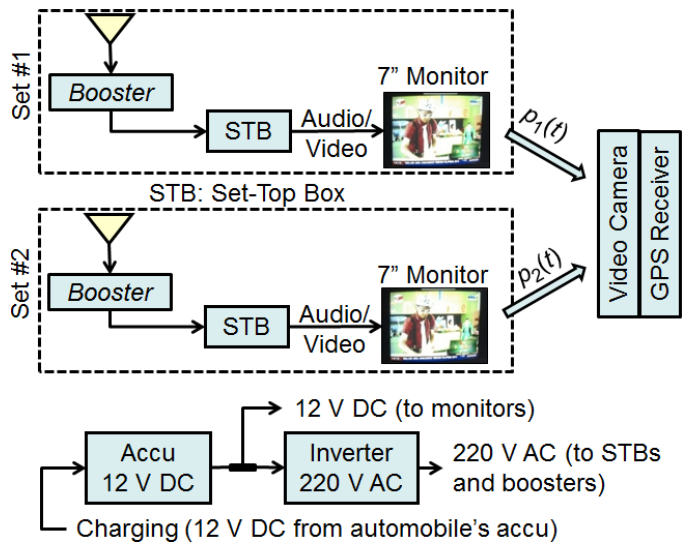

Fig. 6. Detail of the equipment set-up.

The detail of equipment set-up is depicted in Fig. 6. The incabin equipment consisted of two receivers supported by a common electrical power supply system, a common video camera, and an optional GPS (Geo/Global Positioning System) receiver. Two sets of antenna-booster couple were mounted at the outside top of the automobile roof. Each receiver consisted of an STB (set-top box) accompanied by a monitor screen.

Observed channel was number $47(678-686 \mathrm{MHz})$ because it was the only channel being on-air at the time of measurement. Some of transmit parameters detected included: (1) mode: 8K mode, (2) GI: 1/32, and (3) QAM-order: 64.

TABLE I

TRAVELLED ROUTE-SEGMENTS

\begin{tabular}{|c|c|c|c|c|c|c|}
\cline { 2 - 7 } \multicolumn{1}{c|}{} & \multicolumn{7}{c|}{ Route-Segment } \\
\cline { 2 - 7 } \multicolumn{1}{c|}{} & \#a & \#b & \#c & \#d & \#e & \#f \\
\hline Main Orientation & S-N & W-E & S-N & E-W & N-S & W-E \\
\hline Length (km) & 2.36 & 1.42 & 4.44 & 2.37 & 2.63 & 1.5 \\
\hline Duration (s) & 443 & 271 & 974 & 513 & 722 & 498 \\
\hline $\begin{array}{c}\text { Average speed } \\
(\mathbf{k m} / \mathbf{h})\end{array}$ & 19.18 & 18.86 & 16.41 & 16.63 & 13.11 & 10.84 \\
\hline
\end{tabular}

There are six route-segments in Yogyakarta City have been travelled. Besides their traffic density, the shortness of distance between cross-roads (traffic lights) at all route-segments constrained the automobile's speed. Although the streets are not straight lines, their main compass directions are easily identified: East-to-West (E-W), West-to-East (W-E), North-toSouth (N-S), or South-to-North (S-N). The shortest and longest route segments were about $1.29 \mathrm{~km}$ and $4.44 \mathrm{~km}$, respectively. These routes are listed in TABLE I.

Pictures recorded by the camera for each route-segment were processed further as shown in Fig. 7. The upper part of this figure described the physical diagram, whereas its equivalent functional diagram is shown at the lower part.

Each $K T$-second duration video file was converted into a set (series) of $K$ “*.jpg" image files. Such a conversion is equivalent to sample the picture $p_{i}(t)$ with sampling period as long as $T=1$ second. Therefore, the $k$-th image file is the $k$-th time-sample of its associate picture.

STB used in this research produced still picture when it failed to receive. Therefore, the presence of motion can be easily identified by comparing the $k$-th image to its $(k-1)$-th one, where two distinguishable or identical pictures indicate the presence or absence of motion (successful or failed of reception), respectively.
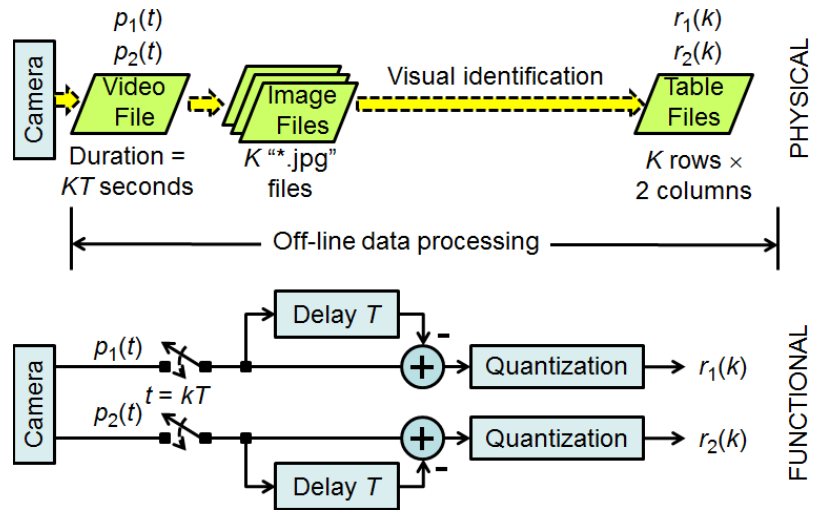

Fig. 7. Off-line data processing of per route-segment recorded picture.

Having been processed as shown Fig. 7, the collected data was summarized in TABLE I. It shows that as many as $K=$ 3.140 time-samples have been collected from the six routes.

TABLE II

Summary OF THE COLlected DATA

\begin{tabular}{|l|c|c|c|c|c|c|l|}
\cline { 2 - 8 } \multicolumn{1}{c|}{} & \multicolumn{7}{c|}{ Route-Segment } \\
\cline { 2 - 9 } \multicolumn{1}{c|}{} & \#a & \#b & \#c & \#d & \# & \#f & Total \\
\hline $\boldsymbol{K}$ & 427 & 259 & 876 & 495 & 640 & 443 & 3140 \\
\hline $\boldsymbol{L}_{\mathbf{1}}$ & 200 & 145 & 216 & 110 & 199 & 186 & 1056 \\
\hline $\boldsymbol{L}_{\mathbf{2}}$ & 127 & 57 & 134 & 77 & 54 & 189 & 638 \\
\hline $\boldsymbol{L}$ & 163.5 & 101 & 175 & 93.5 & 126.5 & 187.5 & 847 \\
\hline $\boldsymbol{C}$ & 116 & 48 & 77 & 39 & 25 & 113 & 418 \\
\hline $\boldsymbol{U}$ & 211 & 154 & 273 & 148 & 228 & 262 & 1276 \\
\hline
\end{tabular}

\section{Single-Antenna System AND CHANNEl CONDITION}

\section{A. Single-Antenna System}

As listed in TABLE II, an antenna gave a better result(s) than the other at a (several) route(s), and otherwise at other route(s). Success-rate of individual antennas and equivalent singleantenna is shown in Fig. 8.

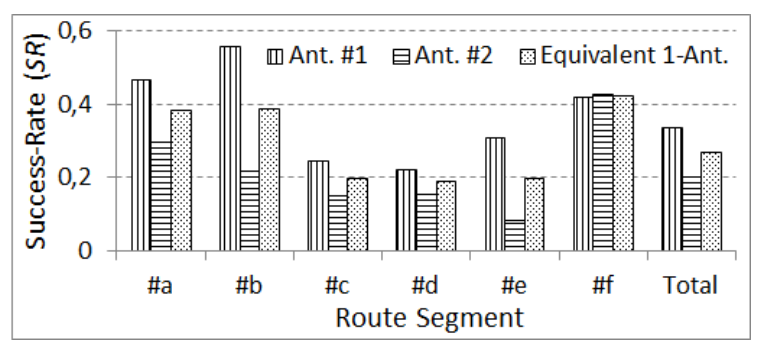

Fig. 8. Success-rates of the single-antenna systems.

In this figure, $S R$ spans approximately from 0.08 (antenna \#2 at route \#e) to 0.56 (antenna \#1 at route \#b). It is low enough and represents that signal quality at those six routes was also not sufficiently good. Such poor coverage might be caused by the surrounding buildings as well as other mobile obstacles in these routes. Antenna \#1 provided a better result (higher $S R$ ) than antenna \#2 at five routes. That antenna \#1 was better than antenna \#2 at majority of the routes is reasonable, because the later was placed (mounted) slight below the former one.

By using only one antenna, its radiation pattern should be as omnidirectional as possible because from which direction the signal arrives is unpredictable. Therefore, increasing the $S R$ can only be done by using a high sensitivity receiving equipment. 


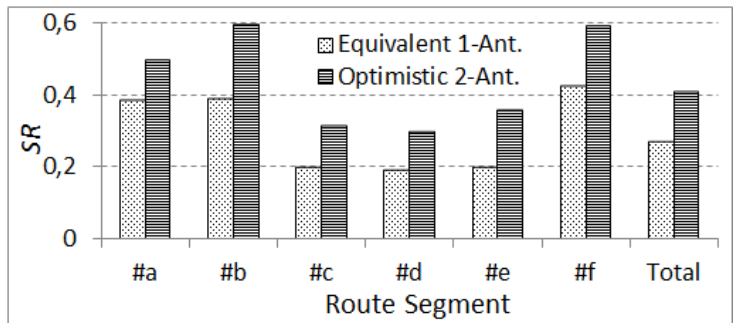

(a)

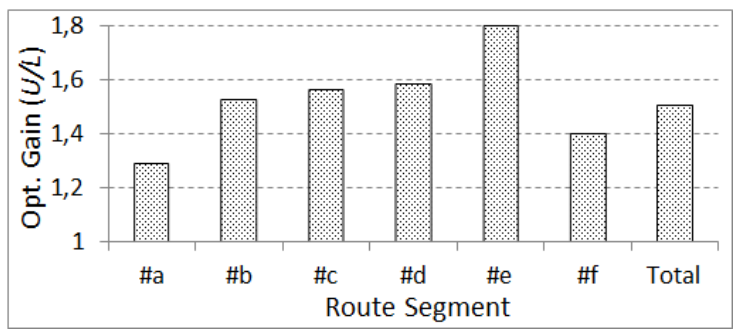

(b)

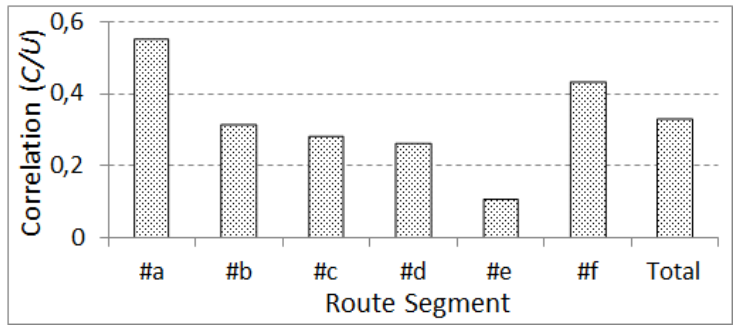

(c)

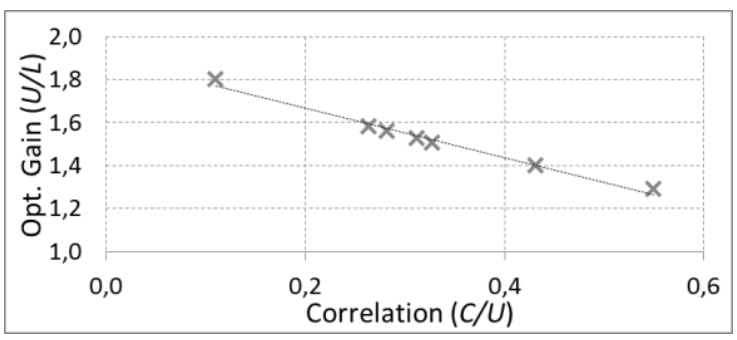

(d)

Fig. 9 Radio channel condition: (a) $S R$ comparison, (b) optimistic-gain, (c) inter-antenna correlation, and (d) relationship between optimistic-gain and correlation.

\section{B. Radio Channel Condition}

The condition of radio channel evaluated using two antennas employed in the proposed method is described in Fig. 9. Fig. 9 (a) shows that the proposed method offered $S R$ increment at all routes with optimistic-gain (Fig. 9 (b)) ranging from 1.29 (route \#a) to 1.80 (route \#e), approximately. This agreed to the correlation (Fig. 9 (c)), which are at the highest at route \#e (about 0.55) and lowest at route \#a (about 0.11), respectively. Relationship between correlation and optimistic-gain is shown in Fig. 9 (d). It shows that the gain decreases when the correlation increases almost linearly.

All antennas should collaboratively cover the whole $360^{\circ}$ azimuth direction and correlation among them should be as low as possible. Therefore, if only two antennas used as in this research, their radiation patterns should be perpendicularly oriented to each other, as illustrated in Fig. 5. Briefly, if $I$ identical antennas are used, horizontal radiation pattern of every antenna should be directional enough, and orientation of two adjacent antenna are separated as far as $360 \%$ to each other.

\section{Performance of the Proposed Method}

\section{A. Reception-Success Improvement}

Collected data summarized in TABLE II was used to simulate the algorithm for $T_{f}=1,2,3,4$, and 5 seconds. Simulation result is summarized in Fig. 10. $S R$ for $T_{f}$ from 1 to 5 seconds is summarized in Fig. 10 (a). Average $S R$ for each route is obtained by averaging $S R$ belongs to the six routes. It is evident that for all of the route-segments and $T_{f}, S R$ was higher than those for the equivalent single-antenna technique $(L)$ although lower than those for the optimistic value $(U)$.

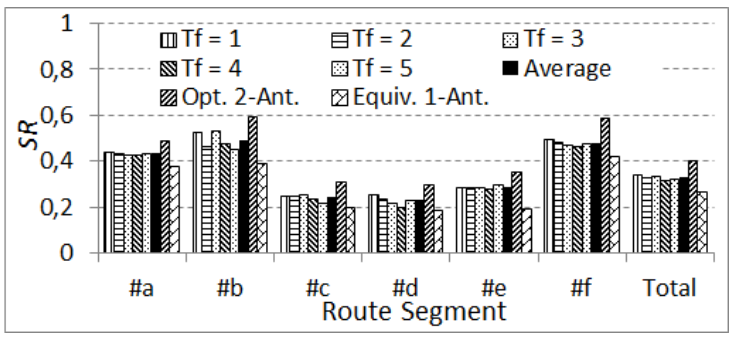

(a)

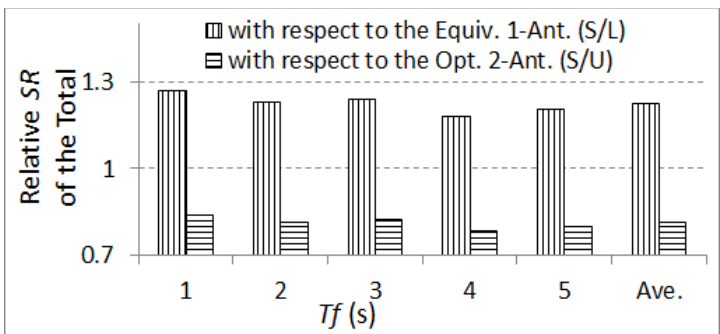

(b)

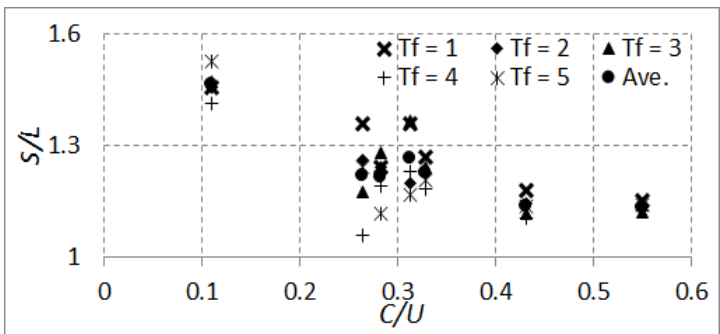

(c)

Fig. 10. Performance of the proposed method: (a) $S R$, (b) Relative $S R$, and (c) relationship between realistic-gain and correlation.

In Fig. 10 (b), the relative $S R$ with respect to $L$ and $U$ for various $T_{f}$ is depicted. For each $T_{f}$ in this figure, $S R$ is the average of six route segments. The best improvement was obtained by $T_{f}=1 \mathrm{~s}$ with $S / L$ and $S / U$ are as high as 1.27 and 0.84 , approximately, whereas $T_{f}=4$ showed the worst one about 1.18 and 0.78 for $S / L$ and $S / U$, respectively.

$S / L$ in Fig. 10 (c) was obtained from a simulation although the simulated data was generated from field-measurement. Therefore, claiming it as the realistic-gain was considered more appropriate rather than the factual-gain. Its trend agreed with the result as shown in Fig. 9 (d).

\section{B. Success-Rate Consistency of the Antennas}

The relative success-rate of antenna \#2 with respect to antenna \#1 when they operated individually as illustrated in Fig. 4 was $L_{2} / L_{1}$. After collaboratively operated as in Fig. 3 (a), 
their ratio of contributed successful-reception became $S_{2} / S_{1}$. Perfectly consistent result should give $S_{2} / S_{1}=L_{2} / L_{1}$, or $\left(S_{2} / S_{1}\right) /\left(L_{2} / L_{1}\right)=1$. Fig. 11 shows that their consistencies were not perfect, and not similar to each other for all routes or $T_{f}$-S. This fact was reasonable because how high an antenna contributes its successful-reception depends not only on its cumulative-duration but also on its time distribution with respect to the others.

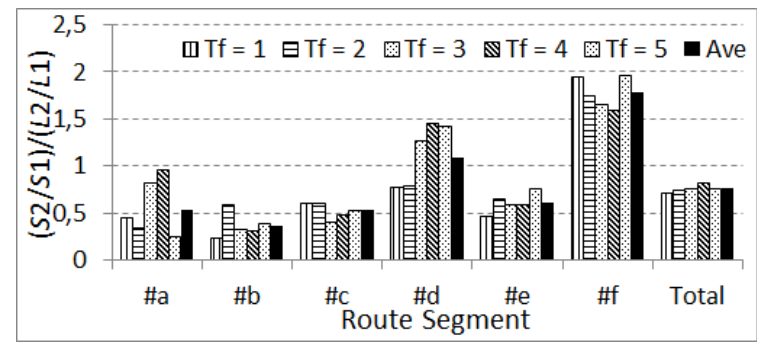

(a)

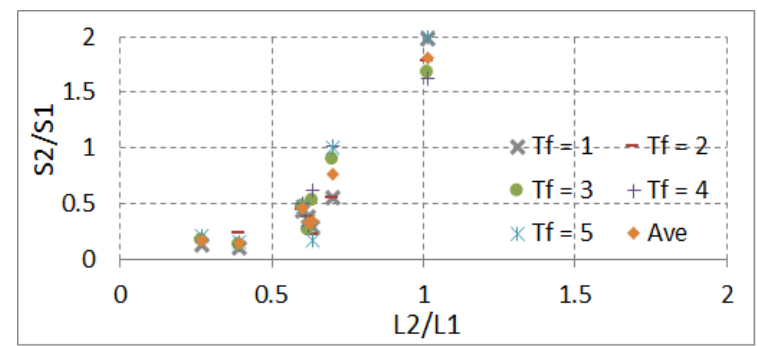

(b)

Fig. 11. Comparison of relative antenna success-rate between collaborative and individual operation: (a) per route-segments, and (b) scatter-plot.

\section{CONCLUSION}

A proposed multiple-antenna method based on recoveredpicture for the DVB-T2 receiver has been evaluated. At any time, signal fed to receiver was selected from antenna being giving a successful-reception. Selected antenna was switchedover to the other immediately after the reception of the being feeding (current selected) antenna continuously failed to exceed a pre-specified tolerable duration. In case that the number of antenna was two, the result of simulation using real data indicated that the proposed method was able to increase the optimistic and realistic reception-success concerning its equivalent single-antenna technique up to about 1.80 and 1.53 times, respectively.

The time delay of TV receiver used needs to be considered in adjusting a parameter of the proposed method. On the other hand, it affects the performance.

\section{REFERENCES}

[1] M. El-Hajjar and L. Hanzo, 'A Survey of Digital Television Broadcast Transmission Techniques', IEEE Commun. Surv. TUTORIALS, vol. 15 , no. 4, pp. 1924-1949, 2013.

[2] I. Eizmendi, M. Velez, D. G'omez-Barquero, J. Morgade, V. BaenaLecuyer, M. Slimani, and J. Zoellne, 'DVB-T2: The Second Generation of Terrestrial Digital Video Broadcasting System', IEEE Trans. Broadcast., vol. 60, no. 2, pp. 258-271, 2014.
[3] F. A. Contreras, E. Pedraza, and D. Gomez-Barquero, 'DVB-T2 Field Trials Results for Portable Indoor Reception in Colombia', in IEEE Latin America Conference on Communications (LATINCOM), 2014, pp. $1-5$.

[4] M. Slimani, J. Robert, P. Schlegel, U. H. Reimers, R. Burow, F Kattanek, R. Pfeffer, and L. Stadelmeier, 'Results of the DVB-T2 Field Trial in Germany', IEEE Trans. Broadcast., vol. 61, no. June, pp. 177194, 2015.

[5] S. Haykin and M. Moher, Modern Wireless Communication. New Jersey: Pearson Education Inc., 2005.

[6] D. H. PHAM, J. GAO, T. TAGATA, H. ASATO, S. HORI, and T. WADA, 'Implementation of Joint Pre-FFT Adaptive Array Antenna and Post-FFT Space Diversity Combining for Mobile ISDB-T Receiver', IEICE Trans. Commun., vol. E91-B, no. No. 1, January, pp. 127-138, 2008.

[7] L. Yang, G. Ren, W. Zhai, and Z. Qiu, 'Beamforming Based Receiver Scheme for DVB-T2 System in High Speed Train Environment', IEEE Trans. Broadcast., vol. 59, no. 1, pp. 146-154, 2013.

[8] N. Aoyama, Young-Cheol, M. Suzuki, M. Okada, and H. Yamamoto, 'A Simple OFDM Diversity Receiver based on Antenna Combining', in IEEE Radio and Wireless Conference, 2004, pp. 291-293.

[9] Y. Kuwahara, R. Fujii, and H. Hatano, 'Novel Combined Diversity Antenna for OFDM', in IEEE Antennas and Propagation Society International Symposium (APSURSI), 2010.

[10] T. Noritomo, M. Okada, and H. Yamamoto, 'A Joint Antenna and PostDFT Combining Diversity Scheme in OFDM Receiver', in IEEE 1st International Symposium on Wireless Communication Systems, 2004, pp. 140-143.

[11] M. Okada, A. Hashizume, and S. Komaki, 'A JOINT FREQUENCY AND SPACE DIVERSITY SCHEME FOR OFDM', in IEEE 50th Vehicular Technology Conference, vol. 5, 1999, pp. 2939-2943.

[12] S. Ben Slimane, 'A Low Complexity Antenna Diversity Receiver for OFDM Based Systems', in IEEE International Conference on Communications (ICC), 2001, pp. 1147-1151.

[13] S. Kang and J. S. Lehnert, 'Receiver diversity scheme for OFDM systems', Electron. Lett., vol. 39, no. 18, pp. 1359-1361, 2003.

[14] B.-W. Kang, O. Nakamura, H. Tomeba, and F. Adachi, 'Performance comparison of Pre-FFT and Post-FFT OFDM Adaptive Antenna Array', in 3rd IEEE VTS Asia Pacific Wireless Communications Symposium, 2006.

[15] D. H. Pham and T. Wada, 'Application of Array Antenna For a Highspeed ISDB-T Reception', in 2nd International Conference on Communications and Electronics (ICCE), 2008, pp. 351-354.

[16] T. Kojiya, T. Wada, T. Iida, Y. Kita, A. Suyama, S. Sakaguchi, H. Asato, H. Mizutani, and A. Shimizu, 'Evaluation of Diversity Combining Systems for Mobile Reception in Digital Terrestrial Television Broadcasting,, in International Conference on IEEE Consumer Electronics (ICCE), 2009, pp. 1-2.

[17] F. W. Vook and K. L. Baum, 'Adaptive Antennas for OFDM', in IEEE 48th Vehicular Technology Conference, Vol. 1, 1998, pp. 606-610.

[18] A. DAMMANN and S. KAISER, 'Transmit/Receive-Antenna Diversity Techniques for OFDM Systems', Eur. Trans. Telecommun., vol. 13, no. No. 5, September-October, pp. 531-538, 2002.

[19] C. Brousseau, F. Nivole, S. Avrillon, and L. Bertel, 'New Diversity Combination Algorithms for DVB-T COFDM Mobile Reception', in 8th International Conference on Communications (COMM), 2010, pp. 319-322.

[20] A. Malcher and P. Falkowski, 'Analog Reconfigurable Circuits', Int. J. Electron. Telecommun., vol. 60, no. 1, pp. 15-26, 2014. 